\title{
Properties of the $\pi$ state induced by impurities in a d-wave superconductor
}

\author{
Grzegorz Litak ${ }^{a}$ Mariusz Krawiec ${ }^{\mathrm{b}}$ \\ ${ }^{a}$ Department of Mechanics, Technical University of Lublin, Nadbystrzycka 36, PL-20-618 Lublin, Poland \\ ${ }^{\mathrm{b}}$ Institute of Physics and Nanotechnology Center, M. Curie-Sktodowska University, Radziszewskiego 10, \\ PL-20-031 Lublin, Poland
}

\begin{abstract}
We study the properties of a quantum impurity embedded in a superconducting host of a d-wave symmetry. The superconductor is described by the extended negative $U$ Hubbard model while the impurity introduces a repulsive interaction to the system around the central site. We discuss the influence of this repulsion on the local properties (density of states, electron pairing and spontaneous current around the impurity) of the superconductor. We show the condition of $\pi$ - like behaviour, defined as two subsystems having a phase difference of $\pi$, in the system by using a proper combination of attractive pairing bond interaction and repulsive one between electrons located at the impurity and nearest neighbour sites.
\end{abstract}

Key words:

d-wave pairing, impurity, proximity effect

PACS: 74.20.-z, 74.20.Rp, 74.62.Dh, 74.45.+c

Impurities in superconductors $(S C)$ has been a subject of intensive theoretical and experimental studies [1/2]3 recently. In case of a nonuniform d-wave superconductor (surfaces, vortices, cracks, twin boundaries or impurities) $\pi$-phase jump in the $S C$ order parameter can be seen [45]. In this case Josephson current becomes negative in contradiction to the usual 0-phase junction. The situation is similar in granular high- $T_{c}$ materials which can likely form network of microscopic $\pi$-junctions [6] between small regions with different phases of the order parameter. In such systems the zero-energy Andreev bound states, zero-bias conductance peaks, paramagnetic Meisner effect and spontaneously generated currents take place [45]. The situation is also possible for paramagnetic impurities with strong on-site Coulomb repulsion are placed in classical $s$-wave superconductor $[7 / 899$. Due to the proximity effect the $S C$ order parameter is created on the impurity site but change its phase by $\pi$.

The purpose of the present work to check if an analogous solution is possible for an impurity with a repulsive interaction embedded in a $d$-wave paired superconductor.

Let us describe the system by the extended negative $U$ Hubbard model [10] with the Hamiltonian:

$H=\sum_{i j \sigma}\left(t_{i j}-\mu \delta_{i j}\right) c_{i \sigma}^{+} c_{j \sigma}+\frac{1}{2} \sum_{i j \sigma} U_{i j} n_{i \sigma} n_{j-\sigma}$

where $i, j$ label sites of a square lattice, $t_{i j}=-t$ is the hopping integral between nearest neighbour sites and $\mu$ - chemical potential. $U_{i j}<0$ describes attraction between electrons with opposite spins occupying neighbour sites $i$ and $j$. The effect of impurity is introduced in one of the lattice sites $i=0$ via repulsive interaction $U_{0 j}>0$.

In the following we shall work in the Hartree-Fock 


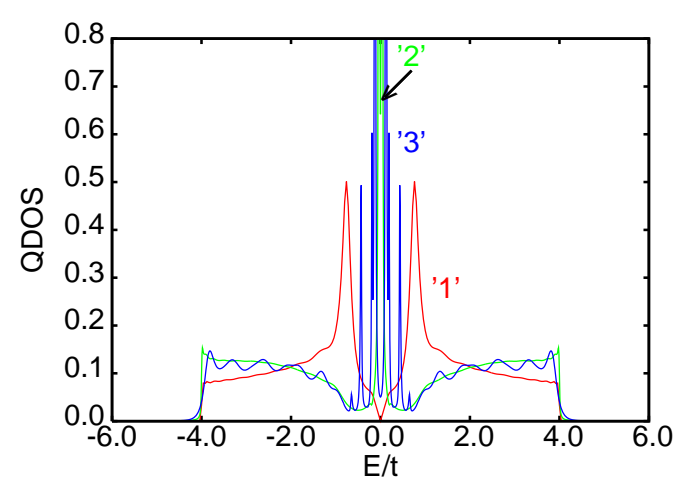

Fig. 1. Quasiparticle density of states $D(E)$ for a pure superconductor '1', $D(E)$ at the impurity site without electric current around the impurity '2' and with current ' 3 '.

approximation dropping the Hartree terms, which means that we have only anomalous part impurity (in a paring potential $\Delta_{i j}$ ) induced disorder in the system. So the corresponding Gorkov equation has the form:

$$
\sum_{j^{\prime}}\left(\begin{array}{c}
(\omega+\mu) \delta_{i j^{\prime}}-t_{i j^{\prime}} ; \Delta_{i j^{\prime}} \\
\Delta_{i j^{\prime}}^{*} ;(\omega+\mu) \delta_{i j^{\prime}}+t_{i j^{\prime}}
\end{array}\right) \hat{G}\left(j^{\prime}, j ; \omega\right)=\delta_{i j} .(2)
$$

In the zero temperature $S C$ order parameter $\Delta_{i j}$ and the total local charge $n_{i}$ are given by selfconsistent relations:

$$
\begin{aligned}
& \Delta_{i j} \equiv U_{i j} \chi_{i j}=-U_{i j} \frac{1}{\pi} \int_{-\infty}^{E_{f}} \mathrm{~d} \omega \operatorname{Im} G^{12}(i, j ; \omega), \\
& n_{i}=-2 \int_{-\infty}^{E_{f}} \mathrm{~d} E D(E)=-\frac{2}{\pi} \int_{-\infty}^{E_{f}} \mathrm{~d} \omega \operatorname{Im} G^{11}(i, i ; \omega),
\end{aligned}
$$

where $E_{f}$ denotes Fermi energy and $D(E)$ defines the quasiparticle density of states. Eqs. (2-3) have been solved self-consistently at the two dimensional lattice using a recursion method [11. Looking for the pairing amplitude at the impurity site $\Delta_{0 j}$, we assumed for simplicity that the surrounding sites may be treated homogeneously as in bulk (clean) superconcuctor. Calculations were performed for interaction parameters: attractive for a bulk $U_{i j} / t=-0.975$, repulsive for an impurity interactions $U_{0 j} / t=3.0$ and a half filled system $(n=1)$. In Fig. 1 the curve '1' corresponds to the quasi-particle density of states for a pure superconductor ' 1 ' while '2' to the local density of states at the impurity site with the $\pi$ phase change in $\Delta_{0 j}$.
The density of states represented by the curve '2' shows two very close peaks located symmetrically near Fermi energy $(E=0)$ signaling bound states within the energy gap and the appearance of the $\pi$ state. Applying an external magnetic field perpendicular to the surface, one can shift those states away the zero energy (curve 3 in Fig. 1). The shifting is proportional to vector potential $\mathbf{A}$ included for a region close to the impurity through a Peierls substitution 1213

$t_{i j} \rightarrow t_{i j} \exp \frac{-\mathrm{i} e}{\hbar} \int_{\mathbf{r}_{\mathbf{i}}}^{\mathbf{r}_{\mathbf{j}}} \mathbf{A}\left(\mathbf{r}^{\prime}\right) \mathrm{d} \mathbf{r}^{\prime}$.

The applied magnetic field generates a current flowing around the impurity.

Those preliminary results will be further checked by a self-consistent calculations with respect to paring amplitude $\Delta_{i j}$, current and the vector potential in order to see if a spontaneous current will be generated in such a system 14.

Acknowledgements: This work has been partially supported by KBN grant No. 2P03B06225. GL would like to thank Max Planck Institute for the Physics of Complex Systems in Dresden for hospitality.

\section{References}

[1] A.V. Balatsky et al., Rev. Mod. Phys. 78, (2006) 373.

[2] J.L. Tallon et al., Phys. Rev. Lett. 79 (1997) 5294.

[3] K. Karpińska et al., Phys. Rev. Lett. 84 (2000) 610.

[4] S. Kashiwaya and Y. Tanaka, Rep. Prog. Phys. 63 (2000) 1641.

[5] T. Löfwander et al., Superconduct. Sci. Technol. 14, (2001) R53.

[6] M. Sigrist and T. M. Rice, Rev. Mod. Phys. 67 (1995) 503.

[7] G. Litak and M. Krawiec, Phys. Stat. Sol. B 242 (2005) 438.

[8] G. Litak and M. Krawiec, Physica B 378-380 (2006), 434.

[9] C.J. Lambert and R. Raimondi, J. Phys. Condens. Matter 10 (1998) 901.

[10] R. Micnas et al., Rev. Mod. Phys. 62 (1991) 113.

[11] G. Litak et al., Physica C 251 (1995) 263.

[12] R.E. Peierls, Z. Physik 80 (1933) 763.

[13] P. Miller, B.L. Gyorffy, J. Phys. Condens. Matter 7 (1995) 5579.

[14] M. Krawiec et al., Phys. Rev. B 66 (2002) 172505. 